Review

\title{
Role of IL-6 inhibitor in treatment of COVID-19-related cytokine release syndrome
}

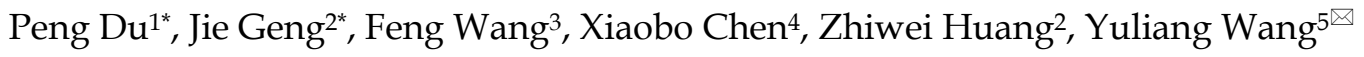 \\ 1. Clinical Laboratory of Emergency Medicine, Tianjin Union Medical Centre, Tianjin, China. \\ 2. Graduate School of Tianjin Medical University, Tianjin, China. \\ 3. Department of Genetics, School of Basic Medical Sciences, Tianjin Medical University, Tianjin, China. \\ 4. Unicell Life Science Development Co., Ltd, Tianjin, China. \\ 5. Department of Clinical Laboratory Medicine, the Second Hospital of Tianjin Medical University, Tianjin Institute of Urology, Tianjin, China. \\ * These authors contributed equally in this manuscript. \\ $凶$ Corresponding author: wangyuliang123@tmu.edu.cn (Y.W.)
}

(1) The author(s). This is an open access article distributed under the terms of the Creative Commons Attribution License (https://creativecommons.org/licenses/by/4.0/). See http://ivyspring.com/terms for full terms and conditions.

Received: 2020.09.22; Accepted: 2020.12.18; Published: 2021.01.21

\begin{abstract}
Cytokine release syndrome (CRS) may be the key factor in the pathology of severe coronavirus disease 2019 (COVID-19). As a major driver in triggering CRS in patients with COVID-19, interleukin-6 (IL-6) appears to be a promising target for therapeutics. The results of inhibiting both trans- and classicalsignaling with marketed IL-6 inhibitors (tocilizumab, siltuximab and sarilumab) in severe COVID-19 patients are effective based on several small studies and case reports thus far. In this review, we described the evidence of the IL-6 response in patients with COVID-19, clarified the pathogenesis of the role of IL-6-mediated CRS in severe COVID-19, and highlighted the rationale for the use of anti-IL-6 agents and key information regarding the potential features of these IL-6 inhibitors in COVID-19 patients.
\end{abstract}

Key words: COVID-19, cytokine release syndrome, interleukin-6, interleukin-6 inhibitor.

\section{Introduction}

On 11 March 2020, the World Health Organization (WHO) announced that novel coronavirus disease 2019 (COVID-19) was an outbreak that was first reported on 8 December 2019 in Hubei Province in China. This pandemic has accounted for $\sim 85$ million confirmed cases and $\sim 1$, 861,005 deaths (as of 7 Jan 2021) in more than 222 countries without even reaching the peak [1]. North America is thus far the most heavily affected area, followed by Europe, Asia and South America. Although showing a lower case-fatality rate, the death toll from COVID-19 is higher than those from severe acute respiratory syndrome (SARS) and Middle East respiratory syndrome (MERS) combined $[2,3]$. The disease-related fatality associated with severe acute respiratory syndrome coronavirus 2 (SARS-CoV-2) infections in humans can be attributed in part to life-threatening hyperinflammation sustained by a cytokine storm that eventually leads to multiple organ failure [4]. This hyperinflammatory response is associated with elevated levels of inflammatory cytokines, including interleukin-6 (IL-6) [5]. Recent data indicate that IL-6 plays an important role in the COVID-19-related cytokine release storm (CRS) [6]. Thus, according to international guidelines, IL-6 inhibitors are recommended as one of the options available to severe or critically ill patients [7]. There has been increased interest in employing the inhibitory agents tocilizumab, siltuximab and sarilumab in COVID-19 patients, with a series of clinical trials being registered and launched in different countries. In addition, the use of anti-IL-6 drugs is associated with an increased risk of some adverse reactions [8]. The use of metalloproteinase 17 (ADAM17) or soluble glycoprotein 130 fused chimera (sgp130Fc) to specifically inhibit pathological IL-6 trans-signaling in patients with severe COVID-19 promises to be a more effective and safer strategy to ameliorate disease states. Therefore, this review aims to summarize the available and latest research 
progress on the association between SARS-CoV-2induced CRS and IL-6 and the development of IL-6 inhibitors.

\section{IL-6: A Double-Edged Sword in Inflammation}

IL-6 can be produced by almost all stromal and immune system cells, including $\mathrm{B}$ lymphocytes, $\mathrm{T}$ lymphocytes, macrophages, monocytes, dendritic cells, mast cells, and other nonlymphocytic cells, such as fibroblasts, endothelial cells, keratinocytes, glomerular mesangial cells and tumor cells. As a multifunctional member of the cytokine network, IL-6 acts as a mediator between pro- and antiinflammatory reactivity by initiating trans- and classical signaling, respectively [9]. In addition, IL-6 is responsible for bridging the gap between the innate and adaptive arms of the immune response [10,11].

\section{Signal Transduction Pathway of IL-6}

The signaling mechanisms employed by IL- 6 are highly complex; therefore, it is often challenging to understand how IL- 6 receptor signaling can create a diverse range of biological responses. There are three main modes of IL-6 signal transduction [9, 12]: classical signal transduction, trans-signal transduction, and a newly reported mechanism called trans-presentation. In the classical signal transduction pathway, IL-6 binds to the membrane-bound IL-6 receptor (mIL-6R) to build a complex [13]. Then, the monomeric complex of IL-6 and mIL-6R associates with ubiquitously expressed transmembrane glycoprotein 130 (gp130), which results in gp130 dimerization. IL-6R exists not only in the transmembrane form but also in a soluble form (sIL-6R). IL-6 binds to these two forms and then interacts with gp130 to initiate intracellular signal transduction and gene expression [14]. sIL-6R can also bind to and activate gp130 on cells that do not carry mIL-6R, thereby enhancing the spectrum of target cells for IL-6, which explains the pleiotropic functions of IL-6. In a healthy state, classical IL-6 receptor signaling is protective and controls various metabolic processes and tissue renewal or regeneration [9]. IL-6 trans-signaling is associated more widely with the regulation of inflammatory processes relevant to disease. A third signal mode, termed IL-6 trans-presentation, is a juxtracrine mechanism of IL-6 signaling that promotes the engagement of dendritic cells with T cells [15], which may lead to priming of Th17 cells in combination with transforming growth factor beta 2 (TGF- $\beta 2$ ), induction of the generation of pathogenic Th17 cells, (chronic) inflammation, and suppression of Treg cells by IL-27. The next process is to activate the Janus kinase/signal transducer and activator of transcription (JAK-STAT) pathway [16]; Additionally, the RAS-RAF [17], SRC-YAP-NOTCH [18] and AKT-PI3K [19] pathways are activated. These processes enable complex biological functions such as proliferation, differentiation, and immune regulation to be realized [20].

\section{Pro- and Anti-inflammatory Effects of IL-6}

IL-6 is the main force that triggers the proinflammatory response during infection. The trans-signaling of IL-6 is significantly positively correlated with the production of proinflammatory cytokines and chemokines [10]. In addition, IL-6 trans-signaling can also stimulate the differentiation of monocytes into macrophages, attract other immune cells, and restrain the function of Treg cells [21]. When infections or tissue injuries occur, IL-6 is promptly produced by monocytes and macrophages and contributes to the removal of infectious agents and restoration of damaged tissues through activation of immune, hematological, and acute-phase responses. Once stress is removed from the host, IL-6 synthesis finishes, but uncontrolled excessive or persistent IL-6 production plays a pathological role in the development of acute severe inflammatory diseases or chronic diseases and cancers [22].

Though often described as a proinflammatory molecule, IL-6 has demonstrated protective properties from a substantial body of evidence. Recent research has indicated a potentially inflammation-suppressing effect of IL-6 in the lungs. The study showed that lung inflammation and injury were more likely to occur in IL-6 knockout (KO) mice exposed to lipopolysaccharide (LPS) than in wild-type (WT) mice [23]. These IL-6 KO mice given exogenous recombinant IL-6 had less severe lung injury and less pulmonary edema than $\mathrm{KO}$ mice not given recombinant IL-6. IL-6 has demonstrated a protective role in the host response to infection. A recent study published in Nature showed that IL-6 KO mice were impaired in response to vaccinia virus, with a 10 1000-fold increase in viral titers in IL-6-deficient animals [24]. These IL-6 KO mice also exhibited increased susceptibility to Listeria monocytogenes infection, possibly because of the reduced bactericidal activity of macrophages. In IL-6 KO mice exposed to inhaled Streptococcus pneumoniae, an increased number of pulmonary $S$. pneumoniae bacterial colonies and reduced survival time relative to WT mice were observed [25]. Moreover, IL-6 enhanced epithelial cell survival and promoted the migration and survival of macrophages. Interestingly, a protective role for IL-6 has been shown in coinfection with influenza virus and S. pneumoniae bacteria. Likewise, IL-6 was elevated during coinfection and 
was the most prominent of all cytokines measured. Together, these data emphasize a vital role for IL-6 in the host immune response to infection. This suggests that blocking IL-6 activity might not promote but diminish host defense against viral or bacterial lung infections.

\section{ADAM17: the Manufacturer of sIL-6R}

The ADAM family member ADAM17 is a type I transmembrane protease that drives the limited proteolysis of over 80 cell membrane-bound cytokines, chemokines, growth factors, adhesion molecules, and their receptors [26]. ADAM-17 is considered to be the key molecule that may explain uncontrolled IL-6 trans-signaling and increased proinflammatory responses during infection. This is because ADAM-17 is the major protein that leads to mIL-6R shedding and, thus, the production of sIL-6R [11]. Apoptosis has been proven to be a natural stimulus in ADAM-17-mediated IL-6R shedding from the surface of neutrophils and thereby contributes to proinflammatory trans-signaling responses [27, 28]. Uncontrolled IL-6 trans-signaling could also be explained by this mechanism, from which a picture of how infection worsens can be envisioned. Therefore, ADAM-17 should be thought of as a potential target molecule for novel antiviral drug discovery that can regulate host reactivity to infection and, in turn, limit or prevent fatal outcomes.

\section{Role for IL-6-mediated CRS of Severe COVID-19}

Huang et al. [6] reported the clinical features and cytokine profile of critically ill patients with COVID-19 in Wuhan, China, and suggested that a cytokine storm, also known as cytokine release syndrome (CRS), could be associated with disease severity. After virus infection, dendritic cells, macrophages, and neutrophils, as the first line of defense, start the immune reaction and affect its type and intensity. Autopsies on patients who died of COVID-19 revealed a high infiltration of macrophages within the area of bronchopneumonia [29]. These macrophages significantly produce IL-6, suggesting that they may be the cause of excessive inflammation in COVID-19 disease [30]. Similarly, in SARS disease, which represents the closest disease to COVID-19 in humans, high production of IL-6 was also previously described. SARS produces even more intense IL-6 than common viral respiratory diseases (e.g., influenza and parainfluenza) [31]. Recent studies have implied the possibility that inflammatory cytokine storms and inflammatory events may be responsible for severe COVID-19 pathology [32]. Thus, IL-6 should not be ignored in the treatment of severe COVID-19.

According to a recent meta-analysis, significantly higher levels of IL-6 in serum are demonstrated to be predictors of the disease severity and prognosis of patients with COVID-19 [33]. Likewise, another meta-analysis indicated that elevated IL-6 levels occur more often in severe and critically ill COVID-19 patients than in mildly ill COVID-19 patients, and they occur more often in patients who die from the disease than in those who survive [34]. This might help clinicians identify critical patients in a timelier and more effective manner.

However, before regarding IL-6-mediated CRS as the pathological driver of severe COVID-19, caution should be warranted. It is noteworthy that COVID-19 patients lack most of the hallmarks of CRS, including hypotension, capillary leak syndrome, and neurotoxicity [35]. In addition, the clinical course of CRS is much more acute than that of COVID-19 [36]. Evidence shows that, compared with 1,000-10,000 $\mathrm{pg} / \mathrm{ml}$ in CRS, serum IL-6 levels are far lower in COVID-19, with peak levels typically less than 100 $\mathrm{pg} / \mathrm{ml}$ in COVID-19 [33, 36].

\section{IL-6 Inhibition as a Strategy for the Treatment of COVID-19-related CRS}

In view of the key role of IL-6 in COVID-19-related CRS, neutralizing antibodies used to treat a number of autoimmune diseases by targeting the exacerbated inflammatory immune response of the host may provide a life-saving approach by preventing cytokine release syndrome in severe cases. Tocilizumab, a specific monoclonal antibody that blocks IL-6, has been recommended for use in severely or critically ill patients with extensive lesions in bilateral lungs and confirmed elevated levels of IL-6 in the Diagnosis and Treatment Protocol for Novel Coronavirus Pneumonia (Trial Version 8) issued by the National Health Commission of China on August 19th, 2020 [37]. Tocilizumab is also mentioned in the NIH treatment guidelines and the WHO interim guidance, both of which cite insufficient clinical data to recommend either for or against the use of these drugs, the same as for other drugs [7, 38]. At present, more IL-6 inhibitors, such as sarilumab and siltuximab, are utilized in the treatment of COVID-19 based on the biology of IL-6.

\section{Tocilizumab (Actemra)}

Tocilizumab is a humanized anti-IL-6R monoclonal antibody. Several cohort studies demonstrated the efficacy of tocilizumab in treating severe cases of COVID-19. A Chinese retrospective 
observational study reported a case series of patients with COVID-19 treated with tocilizumab [39]; 46.7\% of these patients were critically ill. The study authors summarize that tocilizumab might be an applicable treatment with high IL-6. Xu et al [40] reported 21 patients diagnosed with severe or critical COVID-19. Twenty of those 21 patients recovered and were discharged within 2 weeks after tocilizumab therapy, with no adverse drug events. The authors reported that the body temperatures of all patients returned to normal values within the first day after receiving tocilizumab and remained stable thereafter. By the fifth day of treatment, $75 \%$ of patients had a reduced need for oxygen supplementation, and one patient needed no oxygen therapy. In a prospective open, single-arm study, 63 patients with severe cases of COVID-19 were administered tocilizumab either once or twice with doses taken $24 \mathrm{~h}$ apart [41]. In one day, marked reductions in fever were observed in all patients except one, and the levels of C-reactive protein (CRP), ferritin, and D-dimer and the lymphocyte count were improved in most patients. In France, an open-label Phase II RCT (CORIMUNOTOCI, NCT04331808) is being conducted by the public assistance hospitals of Paris [42]. The interim results of this study were released recently through press release. Sixty-five of the 129 patients were randomized to receive tocilizumab $8 \mathrm{mg} / \mathrm{kg}(1-2$ injections) along with standard of care, and 64 received standard of care alone. A significantly lower proportion of the patients in the tocilizumab arm attained the primary outcome of the need for ventilation or death at day 14 . These results showed positive effects of tocilizumab and hinted that IL-6 might be an ideal therapeutic target for COVID-19 patients with CRS.

Another prospective study was conducted in Italy on 100 COVID-19 patients with acute respiratory failure [43]. They received two doses of tocilizumab every $12 \mathrm{~h}$. Within ten days, the respiratory conditions of 77 patients were significantly improved. However, the respiratory function of 23 patients deteriorated, 20 of them died, and three patients experienced serious adverse events. Similarly, a retrospective controlled study supports that tocilizumab is not effective in modifying the 30-day mortality of patients with severe and critical COVID-19 with hyperinflammation [44]. It was recently shown that treatment with tocilizumab resulted in poor outcomes in COVID-19 patients and did not prevent them from developing secondary hemophagocytic lymphohistiocytosis [45]. Genentech, the innovator company of tocilizumab, has also initiated a randomized, double-blind, placebo-controlled Phase III clinical trial (COVACTA, NCT04320615) to evaluate the safety and efficacy of tocilizumab plus standard of care in hospitalized adult patients with severe COVID-19 pneumonia compared with placebo plus standard of care [46]. The trial aims to recruit approximately 330 patients globally. Unfortunately, the COVACTA trial did not meet its primary endpoint of improved clinical status in hospitalized adult patients with severe COVID-19-associated pneumonia. In addition, the key secondary endpoints, which included the difference in patient mortality at week four, were not met; however, there was a positive trend in time to hospital discharge in patients treated with tocilizumab. The COVACTA study did not identify any new safety signals for tocilizumab. More prospective controlled randomized clinical trials are expected to evaluate whether tocilizumab is still considered an anti-inflammatory treatment for COVID-19, and further studies should focus on the optimal timing of treatment. The stage of disease may also affect the efficacy of IL-6 blockade therapy.

\section{Sarilumab (Kevzara)}

Sarilumab is a fully human IgG1 monoclonal antibody that targets both IL-6R and mIL-6R, thus inhibiting both classical and trans-IL-6-mediated inflammatory pathways [47]. A recent study showed that sarilumab has exhibited promising potential in improving the symptoms of COVID-19 patients. The addition of sarilumab to the therapy regimen of eight hospitalized COVID-19-positive patients showed significant improvement in respiratory functions by reducing oxygen demand by $30 \%$. Moreover, early and aggressive intervention in these patients with IL-6 blockers resulted in their discharge after 14 days of hospitalization, and seven out of them tested back negative for COVID-19 [48]. In the USA, the 'Evaluation of the Efficacy and Safety of Sarilumab in Hospitalized Patients With COVID-19' (NCT04315298) was launched, which is a phase II/III, randomized, double-blind, placebo-controlled clinical trial, for 400 patients recruited in March 2020 by Sanofi and Regeneron [49]. On July 2nd, 2020, Regeneron Pharmaceuticals, Inc. (NASDAQ: REGN) and Sanofi announced that the U.S. phase 3 trial of Kevzara ${ }^{\circledR}$ (sarilumab) $400 \mathrm{mg}$ in COVID-19 patients requiring mechanical ventilation did not meet its primary and key secondary endpoints. Minor positive trends were observed in the primary prespecified analysis group that did not reach statistical significance, and these trends were countered by negative trends in a subgroup of critical patients who were not mechanically ventilated at baseline. In the primary analysis group, adverse events were experienced by $80 \%$ of Kevzara patients and $77 \%$ of placebo patients. Serious adverse events that occurred 
in at least $3 \%$ of patients and more frequently among Kevzara patients were multiorgan dysfunction syndrome (6\% Kevzara, $5 \%$ placebo) and hypotension (4\% Kevzara, 3\% placebo). Based on the results, the U.S.-based trial was stopped, including in a second cohort of patients who received a higher dose of Kevzara (800 mg). More studies have since been registered or initiated for assessing sarilumab in the treatment of COVID-19.

\section{Siltuximab}

Siltuximab is a chimeric human-mouse monoclonal antibody against IL-6 for the treatment of patients with multicentric Castleman disease (MCD) approved by the Food and Drug Administration (FDA) [50]. It binds directly to soluble IL-6 and prevents it from acting on the IL-6 receptor to activate the IL-6 signaling pathways. Positive preliminary data from 21 COVID-19 patients admitted with respiratory complications have been reported by one center in Italy (NCT04322188), showing that after intravenous introduction of siltuximab, CRP levels were reduced markedly in most patients. Thirty-three percent of patients showed an improvement in their condition and no longer required ventilatory assistance, $43 \%$ were in a stable condition, and $24 \%$ experienced deterioration [51].

What is potentially important is that the clinical results of therapeutic inhibition of IL-6 with tocilizumab or sarilumab are contradictory at this point, at least inferring that in many cases, IL-6-led CRS may not be the main cause of COVID-19-related morbidity and mortality [51]. This may be partly due to the continued production of IL-6 overcoming the direct targeting effect [52]. The failure of the phase III clinical trials of two IL-6 receptor inhibitors, tocilizumab and sarilumab, in the treatment of severe new coronary pneumonia shows that blocking this pathway may only have some efficacy in the most severe patients. These results suggest that in the treatment of critically ill patients, inhibition of only the IL-6 pathway in CRS may not be enough. It may also be necessary to pay attention to other pathways that cause CRS or to combine treatment with antiviral drugs. Combination therapy from multiple aspects may be a promising strategy for the effective treatment of COVID-19. Moreover, in the case of viral infections such as COVID-19, macrophage activation occurs as a primary response to viral infection [53]. Weakening the innate immune response may reduce off-target toxicity, and in the absence of other virus control (through drug therapy or immune-mediated control alternatives), it may cause more serious viral infections. In addition, IL-6 has significant anti-inflammatory properties. This allows people to question the principle of using IL-6 antagonists as a therapy to reduce inflammation. Studies have also shown that IL-6 performs an important function in the early host immune response to infection. Therefore, IL-6 blockade may not be an effective strategy to treat infection. The elevated level of circulating IL-6 in COVID-19 is usually not higher than the level of patients with autoimmune diseases, which raises questions about whether IL-6 is the causative cytokine in COVID-19 pulmonary or systemic injury. Finally, the clinical use of IL-6 inhibitors in autoimmune conditions increases the risk of serious infections (such as tuberculosis). Other common adverse reactions of anti-IL-6 agents include increased transaminase blood levels and cytopenia [54-56]. These issues should arouse attention; that is, IL-6 inhibitors may be harmful in addition to being unlikely to benefit COVID-19 patients.

\section{The Prospect of Targeting Only sIL-6R-dependent Trans-signaling}

It is noteworthy that trans-signaling via sIL6- $\mathrm{R}$ is believed to act in a rather proinflammatory way via recruitment of mononuclear cells, inhibition of T-cell apoptosis, and inhibition of regulatory T-cell differentiation [21]. If we can block the proinflammatory effect of the trans-signaling pathway and retain the protective effect of classic IL-6 signal transduction, it may be possible to impede the otherwise uncontrolled proinflammatory response of severe SARS-CoV-2 infection. Sgp130Fc and an inhibitor of ADAM-17 may become a new "game changer" to block only the trans-signaling pathway as substitutes for tocilizumab.

Sgp130Fc, a fusion protein of the gp130 receptor with the Fc portion of a human immunoglobulin antibody, selectively inhibits the trans-signaling pathway [57]. Therefore, sgp130Fc is expected to be a potential inhibitor of IL-6 in COVID-19 patients because it can retain the regenerative and anti-inflammatory properties of the IL-6 classic pathway and only block the proinflammatory effects mediated by the trans-signaling pathway [10]. Using sgp130Fc in a murine polymicrobial sepsis model improved survival up to $100 \%$. Evidence shows that using sgp130Fc to block IL-6 trans-signaling is as effective as using a specific monoclonal antibody to completely block IL-6 classic and trans-signaling [58]. Thus, inhibition of only the proinflammatory pathway of IL-6 seems to be a more reasonable choice than inhibition of both pathways.

As we mentioned above, ADAM-17 is mostly responsible for mIL-6R shedding and producing sIL-6R and controls pro- and anti-inflammatory responses to viral antigenic stimuli. Several studies 
have suggested that the pathogenesis mediated by ADAM-17-induced IL-6 trans-signaling is common to inflammatory bowel disease and many respiratory diseases $[59,60]$. What may be inferred from this is that in severe SARS-CoV-2 infection, ADAM-17induced IL-6 trans-signaling eventually interferes with the balance between pro- and anti-inflammatory responses and initiates IL-6 trans-signaling-mediated continuous pro-inflammatory responses. Highly selective and potent ADAM17 inhibitors such as A17pro are expected to be more effective in suppressing disease states related to IL-6 trans-signaling, with fewer adverse effects. Hence, developing a selective inhibitor of ADAM-17 for COVID-19 patients with CRS may be a promising strategy to alleviate inflammatory responses.

\section{Conclusion}

COVID-19 has affected millions of people around the world. Thus, the need to find an effective therapeutic approach for serious viral infections or to reevaluate existing methods is more urgent than ever. Precision medicine is generally considered to have fewer side effects and more prominent effects, but only if we know what components are responsible. IL-6 may be the cause of excessive inflammation in SARS-CoV-2-induced CRS. Inhibiting both trans- and classical signaling with marketed IL-6 inhibitors (tocilizumab, siltuximab and sarilumab) in severe COVID-19 patients has been effective in several small studies and case reports thus far. In addition, we propose that a selective inhibitor of ADAM-17 or sgp130Fc may be a possible antiviral target. It may be necessary to conduct more experimental clinical studies to monitor the safety and efficacy of IL-6 inhibitors because it is urgent to give attention to those effectors that can prove to be "life-saving" treatment options.

\section{Acknowledgments}

This work was supported by the National Natural Science Foundation of China (No. 81470982), Tianjin Municipal Health Bureau Key Project of People's Republic of China (No. 16KG105) and Tianjin Health Industry High-level Talent Selection and Training Project - Jinmen Medical Talents.

\section{Competing Interests}

The authors have declared that no competing interest exists.

\section{References}

1. [Internet] World Health organisation. Coronavirus disease 2019 (COVID-19) pandemic. Accessed 09/09/2020. [Available from: https://www.who.int/ emergencies/diseases/novel-coronavirus-2019.
2. [Internet] World Health Organization. Update 49 - SARS Case Fatality Ratio, Incubation Period. Accessed 07/05/2003. [Available from: https://www.who.int/csr/sars/archive/2003_05_07a/en/.

3. [Internet] World Health Organization, Middle East Respiratory Syndrome Coronavirus (MERS-CoV). [Available from: https://www.who.int/ emergencies/mers-cov/en/.

4. Wang YL, Wang F, Geng J. Cytokine and cytokine storm. Tianjin Med J 2020; 48(6): 494-499.

5. Tanaka T, Narazaki M, Kishimoto T. Immunotherapeutic implications of IL-6 blockade for cytokine storm. Immunotherapy 2016; 8(8): 959-970.

6. Huang C, Wang Y, Li X, et al. Clinical features of patients infected with 2019 novel coronavirus in Wuhan, China. Lancet 2020; 395(10223): 497-506.

7. [Internet] Clinical management of COVID-19 INTERIM GUIDANCE. Accessed 28/05/2020. [Available at: https://apps.who.int/iris/ bitstream/handle/10665/332196/WHO-2019-nCoV-clinical-2020.5-eng.pdf?s equence $=1 \&$ is Allowed $=\mathrm{y}$.

8. Crisafulli S, Isgrò V, La Corte L, Atzeni F, Trifirò G. Potential Role of Anti-interleukin (IL)-6 Drugs in the Treatment of COVID-19: Rationale, Clinical Evidence and Risks. BioDrugs 2020; 34(4): 415-422.

9. Hunter CA, Jones SA. Corrigendum: IL-6 as a keystone cytokine in health and disease. Nat Immunol 2017; 18(11): 1271.

10. Scheller J, Chalaris A, Schmidt-Arras D, Rose-John S. The pro- and anti-inflammatory properties of the cytokine interleukin-6. Biochim Biophys Acta 2011; 1813(5): 878-888.

11. Commins SP, Borish L, Steinke JW. Immunologic messenger molecules: cytokines, interferons, and chemokines. J Allergy Clin Immunol 2010; 125(2 Suppl 2): S53-72.

12. Uciechowski P, Dempke WCM. Interleukin-6: A Masterplayer in the Cytokine Network. Oncology 2020; 98(3): 131-137.

13. Baran P, Hansen S, Waetzig GH, et al. The balance of interleukin (IL)-6, IL-6 soluble IL-6 receptor (sIL-6R), and IL-6 sIL-6R sgp130 complexes allows simultaneous classic and trans-signaling. J Biol Chem 2018; 293(18): 6762-6775.

14. Zhang C, Wu Z, Li JW, Zhao H, Wang GQ. Cytokine release syndrome in severe COVID-19: interleukin-6 receptor antagonist tocilizumab may be the key to reduce mortality. Int J Antimicrob Agents 2020; 55(5): 105954

15. Heink S, Yogev N, Garbers C, et al. Trans-presentation of IL-6 by dendritic cells is required for the priming of pathogenic TH17 cells. Nat Immunol 2017; 18(1): 74-85.

16. Johnson DE, O'Keefe RA, Grandis JR. Targeting the IL-6/JAK/STAT3 signalling axis in cancer. Nat Rev Clin Oncol 2018; 15(4): 234-248.

17. Villarino AV, Kanno Y, O'Shea JJ. Mechanisms and consequences of Jak-STAT signaling in the immune system. Nat Immunol 2017; 18(4): 374-384.

18. Taniguchi K, Wu LW, Grivennikov SI, et al. A gp130-Src-YAP module links inflammation to epithelial regeneration. Nature 2015; 519(7541): 57-62.

19. Yamada O, Ozaki $K$, Akiyama $M$, Kawauchi K. JAK-STAT and JAK-PI3K-mTORC1 pathways regulate telomerase transcriptionally and posttranslationally in ATL cells. Mol Cancer Ther 2012; 11(5): 1112-1121.

20. Jones SA, Jenkins BJ. Recent insights into targeting the IL- 6 cytokine family in inflammatory diseases and cancer. Nat Rev Immunol 2018; 18(12): 773-789.

21. Neurath MF, Finotto S. IL-6 signaling in autoimmunity, chronic inflammation and inflammation-associated cancer. Cytokine Growth Factor Rev 2011; 22(2): 83-89.

22. Tanaka T, Narazaki M, Masuda K, Kishimoto T. Regulation of IL-6 in Immunity and Diseases. Adv Exp Med Biol 2016; 941: 79-88.

23. Voiriot G, Razazi K, Amsellem V, Tran Van Nhieu J, Abid S, Adnot S, Mekontso Dessap A, Maitre B. Interleukin-6 displays lung anti-inflammatory properties and exerts protective hemodynamic effects in a double-hit murine acute lung injury. Respir Res 2017; 18(1): 64.

24. Kopf M, Baumann H, Freer G, Freudenberg M, Lamers M, Kishimoto $T$, Zinkernagel R, Bluethmann H, Köhler G. Impaired immune and acute-phase responses in interleukin-6-deficient mice. Nature 1994; 368(6469): 339-342.

25. van der Poll T, Keogh CV, Guirao X, Buurman WA, Kopf M, Lowry SF. Interleukin-6 gene-deficient mice show impaired defense against pneumococcal pneumonia. J Infect Dis 1997; 176(2): 439-444.

26. Zunke F, Rose-John S. The shedding protease ADAM17: Physiology and pathophysiology. Biochim Biophys Acta Mol Cell Res 2017; 1864(11 Pt B): 2059-2070.

27. Mahmud-Al-Rafat A, Majumder A, Taufiqur Rahman KM, Mahedi Hasan AM, Didarul Islam KM, Taylor-Robinson AW, Billah MM. Decoding the enigma of antiviral crisis: Does one target molecule regulate all? Cytokine 2019; 115: 13-23.

28. Wang Y, Robertson JD, Walcheck B. Different signaling pathways stimulate a disintegrin and metalloprotease-17 (ADAM17) in neutrophils during apoptosis and activation. J Biol Chem 2011; 286(45): 38980-38988.

29. Barton LM, Duval EJ, Stroberg E, Ghosh S, Mukhopadhyay S. COVID-19 Autopsies, Oklahoma, USA. Am J Clin Pathol 2020; 153(6): 725-733.

30. Park MD. Macrophages: a Trojan horse in COVID-19? Nat Rev Immunol 2020; 20(6): 351 .

31. Paces J, Strizova Z, Smrz D, Cerny J. COVID-19 and the immune system. Physiol Res 2020; 69(3): 379-388.

32. Zumla A, Hui DS, Azhar EI, Memish ZA, Maeurer M. Reducing mortality from 2019-nCoV: host-directed therapies should be an option. Lancet 2020; 395(10224): e35-e36. 
33. Chen L, Liu HG, Liu W, Liu J, Liu K, Shang J, Deng Y, Wei S. [Analysis of clinical features of 29 patients with 2019 novel coronavirus pneumonia]. Zhonghua Jie He He Hu Xi Za Zhi. 2020; 43(3): E005.

34. Zhu J, Pang J, Ji P, Zhong Z, Li H, Li B, Zhang J. Elevated interleukin-6 is associated with severity of COVID-19: a meta-analysis. J Med Virol 2020; 29:10.

35. Hay KA, Hanafi LA, Li D, et al. Kinetics and biomarkers of severe cytokine release syndrome after CD19 chimeric antigen receptor-modified T-cell therapy. Blood 2017; 130(21): 2295-2306.

36. Neelapu SS, Tummala S, Kebriaei P, et al. Chimeric antigen receptor T-cell therapy - assessment and management of toxicities. Nat Rev Clin Oncol 2018; 15(1): 47-62.

37. [Internet] National Health Commission and National Administration of Traditional Chinese Medicine. Diagnosis and treatment protocol for novel coronavirus pneumonia (Trial Version 7). Accessed 19/08/2020. [Available from: http://www.nhc.gov.cn/yzygj/s7653p/202008/0a7bdf12bd4b46e5bd 28ca7f9a7f5e5a.shtml

38. [Internet] National Institutes of Health. Coronavirus disease 2019 (COVID- 19) treatment guidelines. Accessed 24/04/2020. [Available from: https://www. covid19treatmentguidelines.nih.gov/.

39. Luo P, Liu Y, Oiu L, Liu X, Liu D, Li J. Tocilizumab treatment in COVID-19: A single center experience. J Med Virol 2020; 92(7): 814-818.

40. Xu X, Han M, Li T, et al. Effective treatment of severe COVID-19 patients with tocilizumab. Proc Natl Acad Sci U S A 2020; 117(20): 10970-10975.

41. Sciascia S, Aprà F, Baffa $\mathrm{A}$, Baldovino $\mathrm{S}$, et al. Pilot prospective open, single-arm multicentre study on off-label use of tocilizumab in patients with severe COVID-19. Clin Exp Rheumatol 2020; 38(3): 529-532.

42. [Internet] CORIMUNO-19-Tocilizumab Trial-TOCI (CORIMUNO-TOCI) (CORIMUNO-TOC). Accessed 08/05/2020. [Available from: https://clinicaltrials.gov/ct2/show/NCT04331808.

43. Toniati P, Piva S, Cattalini M, et al. Tocilizumab for the treatment of severe COVID-19 pneumonia with hyperinflammatory syndrome and acute respiratory failure: A single center study of 100 patients in Brescia, Italy. Autoimmun Rev 2020; 19(7): 102568

44. Canziani LM, Trovati S, Brunetta E, et al. Interleukin-6 receptor blocking with intravenous tocilizumab in COVID-19 severe acute respiratory distress syndrome: A retrospective case-control survival analysis of 128 patients. J Autoimmun. 2020; 102511

45. Radbel J, Narayanan N, Bhatt PJ. Use of Tocilizumab for COVID-19-Induced Cytokine Release Syndrome: A Cautionary Case Report. Chest 2020; 158(1): e15-e19.

46. [Internet] Roche provides an update on the phase III COVACTA trial of Actemra/Roactenra in hospitalised patients with severe COVID 19 associated pneumonia. Accessed 29/07/2020. [Available from: https://pipelinereview. com/index.php/2020072975429/Antibodies/Genentech-Provides-an-Updateon-the-Phase-III-COVACTA-Trial-of-Actemra-in-Hospitalized-Patients-WithSevere-COVID-19-Associated-Pneumonia.html

47. Raimondo MG, Biggioggero M, Crotti C, Becciolini A, Favalli EG. Profile of sarilumab and its potential in the treatment of rheumatoid arthritis. Drug Des Devel Ther 2017; 11: 1593-1603.

48. Benucci M, Giannasi G, Cecchini P, et al. COVID-19 pneumonia treated with Sarilumab: A clinical series of eight patients. J Med Virol. 2020; 10.1002/jmv.26062. Online ahead of print

49. [Internet] Regeneron and Sanofi Provide Update on Kevzara ${ }^{\circledR}$ (sarilumab) Phase 3 U.S. Trial in COVID-19 Patients. Accessed 02/07/2020. [Available from: https://www.prnewswire.com/news-releases/regeneron-and-sanofiprovide-update-on-kevzara-sarilumab-phase-3-us-trial-in-covid-19-patients-3 01087849 htm

50. Deisseroth A, Ko CW, Nie L, et al. FDA approval: siltuximab for the treatment of patients with multicentric Castleman disease. Clin Cancer Res 2015; 21(5): $950-4$.

51. Gritti G, Raimondi F, Ripamonti D, et al. IL-6 signalling pathway inactivation with siltuximab in patients with COVID-19 respiratory failure: an observational cohort study. medRxiv (2020) Accessed 01/04/2020. [Available from: https://doi.org/10.1101/2020.04.01.20048561

52. Xu Y, Li X, Zhu B, et al. Characteristics of pediatric SARS-CoV-2 infection and potential evidence for persistent fecal viral shedding. Nat Med 2020; 26(4): 502-505.

53. Tate MD, Ong JDH, Dowling JK, et al. Reassessing the role of the NLRP3 inflammasome during pathogenic influenza A virus infection via temporal inhibition. Sci Rep 2016; 6: 27912.

54. [Internet] European Medicines Agency. Tocilizumab: summary of product characteristics. Accessed 26/05/2020. [Available from: https://www.ema. europa.eu/en/documents/product-information/roactemra-epar-product-inf ormation en.pdf.

55. [Internet] European Medicines Agency. Sarilumab: summary of product

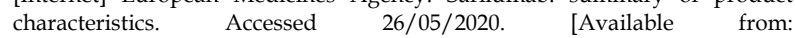
https://www.ema.europa.eu/en/documents/product-information/kevzaraepar-product-information en.pdf.

56. [Internet] European Medicines Agency. Siltuximab: summary of product

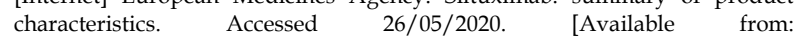
https://www.ema.europa.eu/en/documents/product-information/sylvantepar-product-information_en.pdf.

57. Rose-John S. The Soluble Interleukin 6 Receptor: Advanced Therapeutic Options in Inflammation. Clin Pharmacol Ther 2017; 102(4): 591-598.
58. Barkhausen $T$, Tschernig $T$, Rosenstiel $P$, et al Selective blockade of interleukin-6 trans-signaling improves survival in a murine polymicrobial sepsis model. Crit Care Med 2011; 39(6): 1407-1413.

59. Berry MA, Hargadon B, Shelley M, et al. Evidence of a role of tumor necrosis factor alpha in refractory asthma. N Engl J Med 2006; 354(7): 697-708.

60. Arribas J, Esselens C. ADAM17 as a therapeutic target in multiple diseases. Curr Pharm Des 2009; 15(20): 2319-2335. 